\title{
Activity Monitoring and Unusual Activity Detection for Elderly Homes
}

\author{
Liz George M \\ Computer Science and Engineering, \\ Sahrdaya College of Engineering and Technology, \\ Thrissur 680684, Kerala, India. \\ Marsha Mariya Kappan \\ Computer Science and Engineering, \\ Sahrdaya College of Engineering and Technology, \\ Thrissur,680684, Kerala, India.
}

\author{
Dr.Arun Thomas \\ Associate Prof. \\ Computer Science and Engineering, \\ Sahrdaya College of Engineering and Technology, \\ Thrissur, 680684, Kerala, India. \\ Judith Tony \\ Computer Science and Engineering, \\ Sahrdaya College of Engineering and Technology, \\ Thrissur, 680684, Kerala, India.
}

\author{
Maria Joy \\ Computer Science and Engineering, \\ Sahrdaya College of Engineering and Technology, \\ Thrissur, 680684, Kerala, India.
}

\begin{abstract}
The number of older people in different countries are constantly increasing. Most of this people prefer to live independently. Falls may lead to serious injuries and may even cause death of people. As a solution to this problem it is essential to develop a fall detection system. The objective of this project is to identify and detect unusual activity for an elderly person. Individuals spend the majority of their time in their home or workplace and many feels that these places are their sanctuaries. The information about the person is stored in a database. So in an emergency situation the neighbor can go through the details of the affected person and he/she can refer all the details about the affected person. A camera is continuously capturing the video of the bedridden person. Machine learning techniques use the information to identify and reason about normal behavior in terms of recognized and forecasted activities. Once the abnormal behavior is identified as a threat, a message is sent to the neighbor or corresponding authorities. In most emergency cases, the elderly patient seek in-patient care, which is very expensive and can be a serious financial burden on the patient if the hospital stay is prolonged, and it won't be affordable for everyone. The proposed work allows people to remain in their comfortable home environment rather than inexpensive and limited nursing homes or hospitals, ensuring maximum independence to the occupants. Therefore, an affordable and comprehensive healthcare solution with minimal workforce have much importance for longterm health management and population. We make use of Artificial Intelligence, Machine Learning, and computer vision.
\end{abstract}

Keywords:- Activities of Daily Living (ADL), Support Vector Machine (SVM), Red Green Blue (RGB), Hidden Markov Models (HMM), Sensor of Movements (SoM), Remote Telecare Center (RTC), Decision and Analysis Device (DAD), Human Activity Recognition (HAR).

\section{INTRODUCTION}

In most of our countries, elderly people represent the fastest growing segment of the population, and this trend will increase over the next years. At the same time, the Public Health Services institutions have to face budget restrictions and they have increasing pressure to limit costs. About $30 \%$ of elderly people fall once a year at least, representing $75 \%$ of the victims of falls. The fall event is responsible for $70 \%$ of accidental deaths in persons aged $75+$, and for increasing the level of fear, anxiety or depression leading to the reduction of the day to day activity. These observations have encouraged the development of fall detection systems to detect or even prevent a fall event and to ensure a rapid and efficient help when such an event occurs . But very few fall detection systems are commercially available today, due to lack of reliability, lack of easiness to install and use, or because people did not accept a system found too intrusive or expensive for instance. The greater part of elderly suffer from various chronic diseases and other serious problems of aging. Aging is often related to a functional decline of physical and cognitive abilities, especially in individuals who suffer from diseases such as Alzheimer's disease and dementia. Monitoring the patient's activities of daily living (ADLs) is essential to (a) Detects anomalies and prompts them, (b) Assist the independent living of older adults (c) The diagnosis of diseases and health problems. Formal paid care services offered by hospitals or caregivers, or elderly care centers are expensive and thus are still out of reach for a large section of the elderly population living under constrained or fixed budget conditions. The elderly may require frequent, immediate medical intervention and care, which may otherwise result into fatal consequences. So activity monitoring in a home platform, allows people to remain in their comfortable home environment rather than in expensive and limited nursing homes or hospitals, ensuring maximum independence to the occupants. 


\section{PROBLEM DEFINITION}

Aging is often related to the functional decline of physical and cognitive abilities. Most risk is among individuals suffering from major diseases, fully paralyzed or half paralyzed, while most of the elderly people live alone. People find it difficult to manage their profession along with taking care them. Nowadays a number of methods prevail such as context aware systems like video, surveillance systems, video, microphones, floor sensors, pressure sensors and wearable device-based systems. The major drawback of these systems are, especially in context aware systems is that they compromise users privacy, also they have high initial costa and are liable to external events. Video camera for surveillance is restricted in some countries. The second major category of alternatives is using wearable devices. Majority of them use accelerometer measuring the acceleration changes. The drawback lies where by relying completely only on wearable devices, elderly find it irritating and discomfort on wearing them. Hence a better solution with efficient functionality is to be implemented. People with disabilities, due to their limited mobility and independence, are often deprived of their regular healthcare needs. Furthermore, chronic diseases and conditions such as heart disease, stroke, cancer, and diabetes are among the most common health problems in adults. Therefore, it is no wonder that the demand for healthcare services increases with the increasing average life expectancy of the population. However, the cost associated with present-day healthcare services continues to rise due to the ever-rising prices of prescription drugs, diagnostic tools and in-clinic care.

\section{ARCHITECTURE}

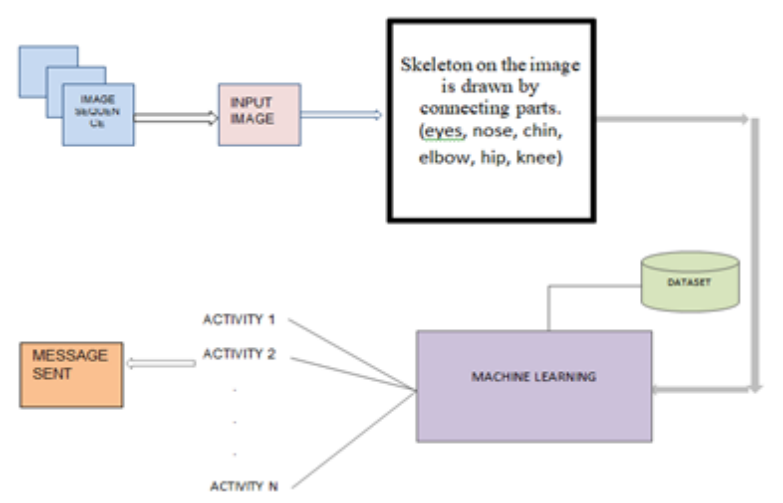

Fig 1:- Architecture

Sequences of images are collected from video capturing using HD camera. Images are captured frame by frame. Within the frame only the silhouette is taken eliminating all the possible background. Hence it is easy to capture only the body position of the person and to identify the activity. From the image sequences the input image is selected for input to the Artificial Neural Network. Skelton is drawn by connecting parts. Openpose algorithm works on parts and pairs. A body part is an element of the body, like neck, left shoulder or right hip. The method works with calculating the distances between each part. Hence for each action the distance measurements changes. This change is considered for predicting various actions. The dataset is pretrained.The dataset used for training consists of frames where videos corresponding to each action are divided into a number of images. 9 actions are trained wave, stand, punch, kick,sit,walk, run, jump, squat. The total video lengths are about 20 minutes, containing more than 10000 video frames recorded at 10 frames per second. Finally when an action is detected the action together with its score is sent as an email to the corresponding persons.

\section{IMPLEMENTATION}

Openpose represents the first real time multi person system to jointly detect human body, hand, facial and foot keypoints. Openpose is a library for real-time multi-person keypoint detection and multi-threading written in $\mathrm{C}++$ using opencv and caffe. The openpose library is built upon a neural network and has been developed by carnegie mellon university with astounding help of COCO and MPII datasets. Openpose algorithm works on parts and pairs. A body part is an element of the body, like neck, left shoulder or right hip. 'Openpose', human pose estimation algorithm, have been implemented using tensorflow. It also provides several variants that have some changes to the network structure for real-time processing on the CPU or low-power embedded devices.

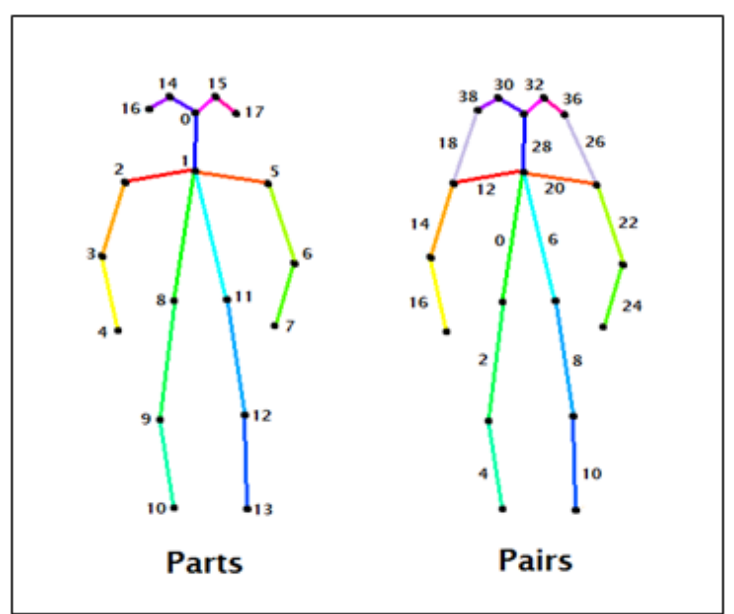

Fig 2:- Human Body Skelton Representation

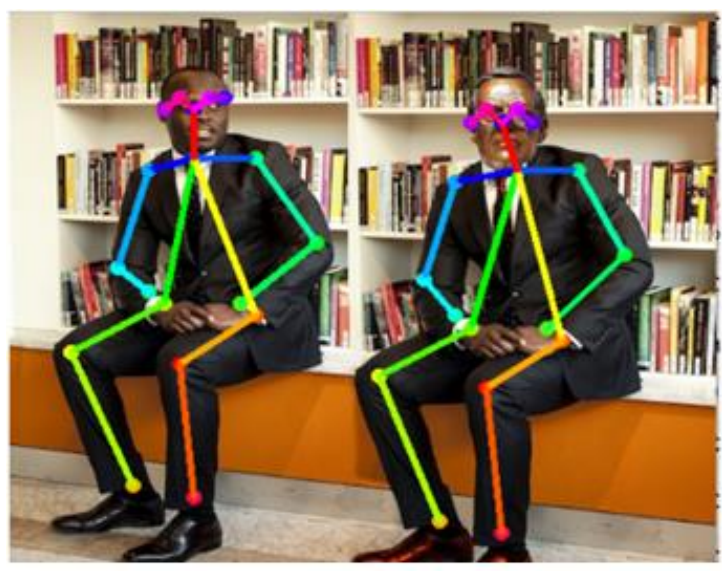

Fig 3:- Recognition of skeleton from human image 


\section{Training:}

The dataset used for training consists of frames where videos corresponding to each actions are divided into a number of images. 9 actions are trained wave, stand, punch, kick,sit,walk, run, jump, squat . The total video lengths are about 20 mins, containing more than 10000 video frames recorded at 10 frames per second. Mobile net thin model of tensorflow is used for training purpose. MobileNets are small, low-latency, low-power models parameterized to meet the resource constraints of a variety of use cases. They can be built upon for classification, detection, embedding and segmentation similar to how other popular large scale models, such as inception is used. Output obtained after training the dataset is a pickle file.

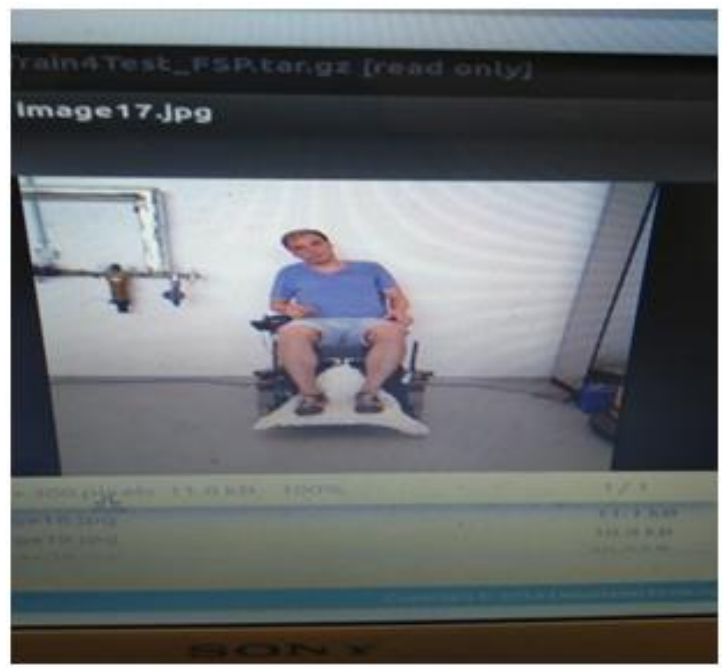

Fig 4:- Training image 1

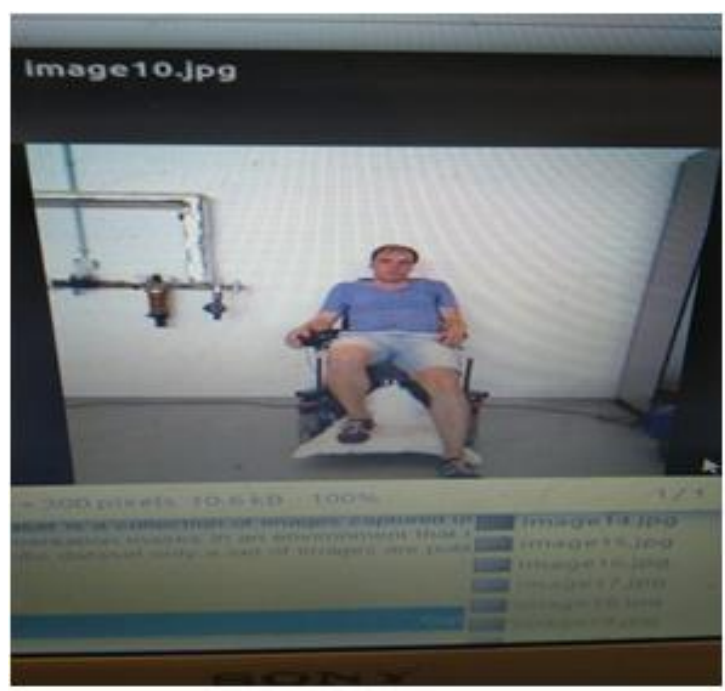

Fig 5:- Training image 2

\section{Testing:}

A tensorflow graph file known as .pb file is used for drawing graphs. Can be done by taking input either from the web cam or direct by inserting image. Skeleton image for each input image frame is found out. This is obtained by interconnecting the specific regions of the body like nose, eyes, chin, shoulder, hip, elbow, knees, Here tf-poseestimation is used. On testing with input from web cam or image we obtain a skeleton structure equivalent to the image along with the action predicted and the score indicating the accuracy. Also, a message indicating the action taken place is send via email by using SMTP module.

Results can be tested in 2 ways:

- By live capturing using webcam.

- By giving an image as input.

- By live capturing using webcam:

In this case the webcam is turned on and video is captured continuously. In Fig.6 the predicted pose is sitting, and in Fig. 7 the predicted pose is upright. Depending on the clarity of camera it will also predict the scene also as in Fig.6 and Fig. 7.

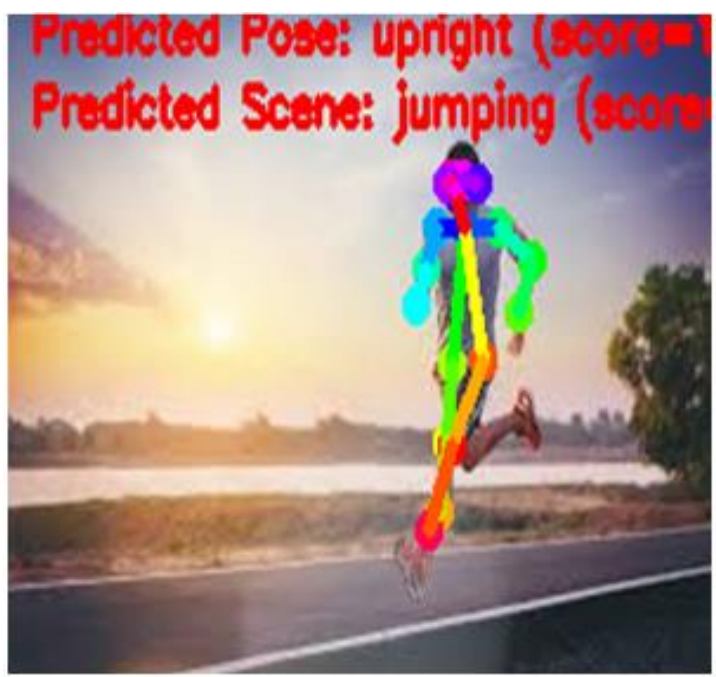

Fig 6:- Predicted pose: Upright. Predicted scene: jumping.

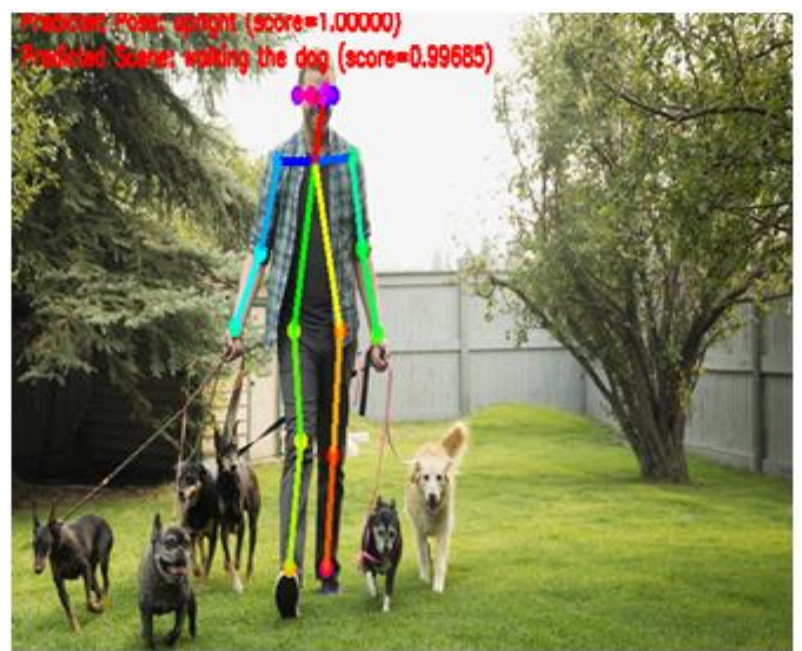

Fig.7:- Predicted pose: Upright

Predicted scene: walking with dog

- By giving an image as input.

Here images are given as input. The pose and scene together with scores are predicted. Also an output image is formed in which skeleton is drawn. 


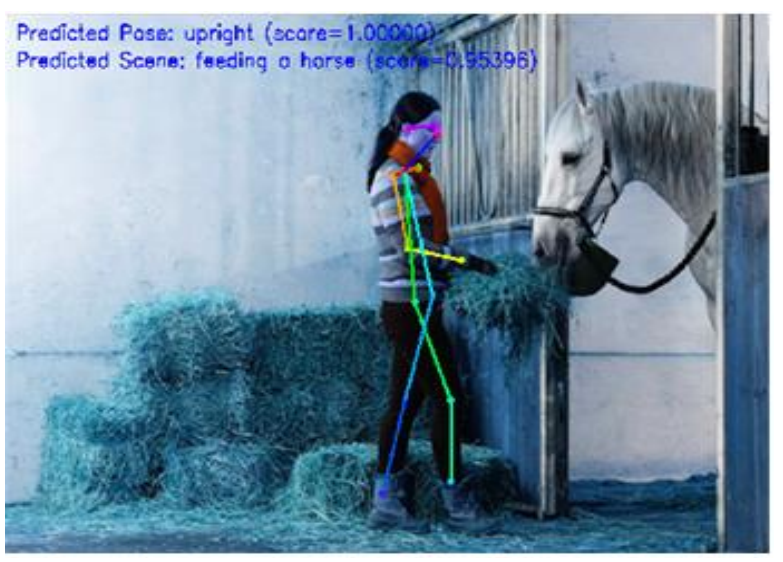

Fig 8:- Predicted pose: Upright.

Predicted scene: feeding a horse.

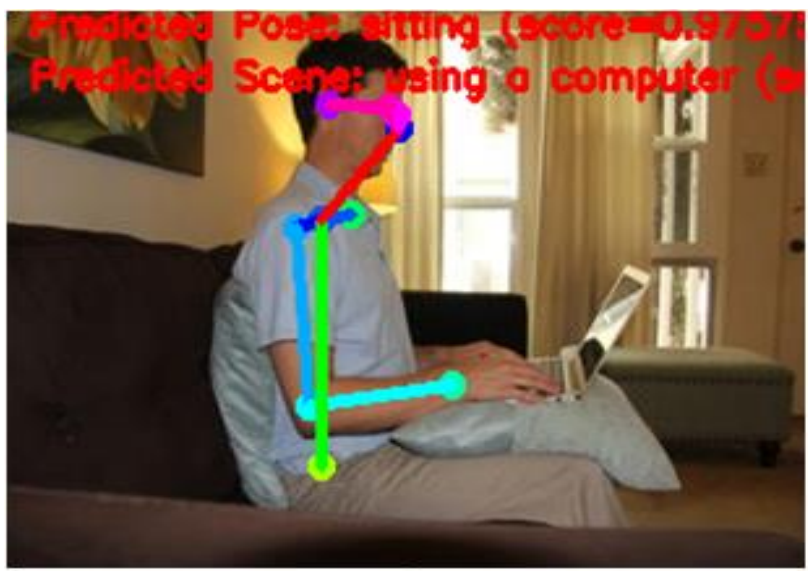

Fig 9:- Predicted pose: Sitting.

Predicted scene: Using a computer.

\section{Message Passing:}

When an action is detected then the action together with its score is sent to the corresponding mail id's.

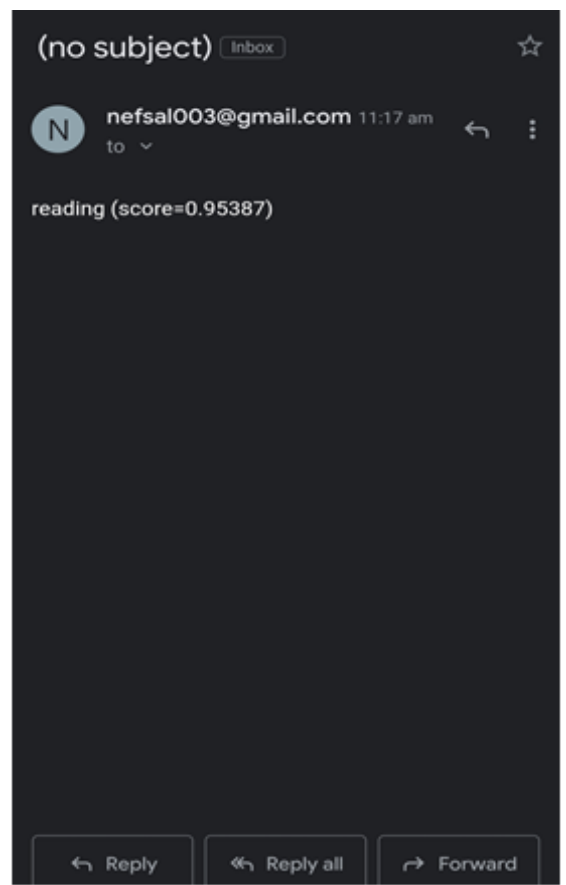

Fig 10:- Message1 sent

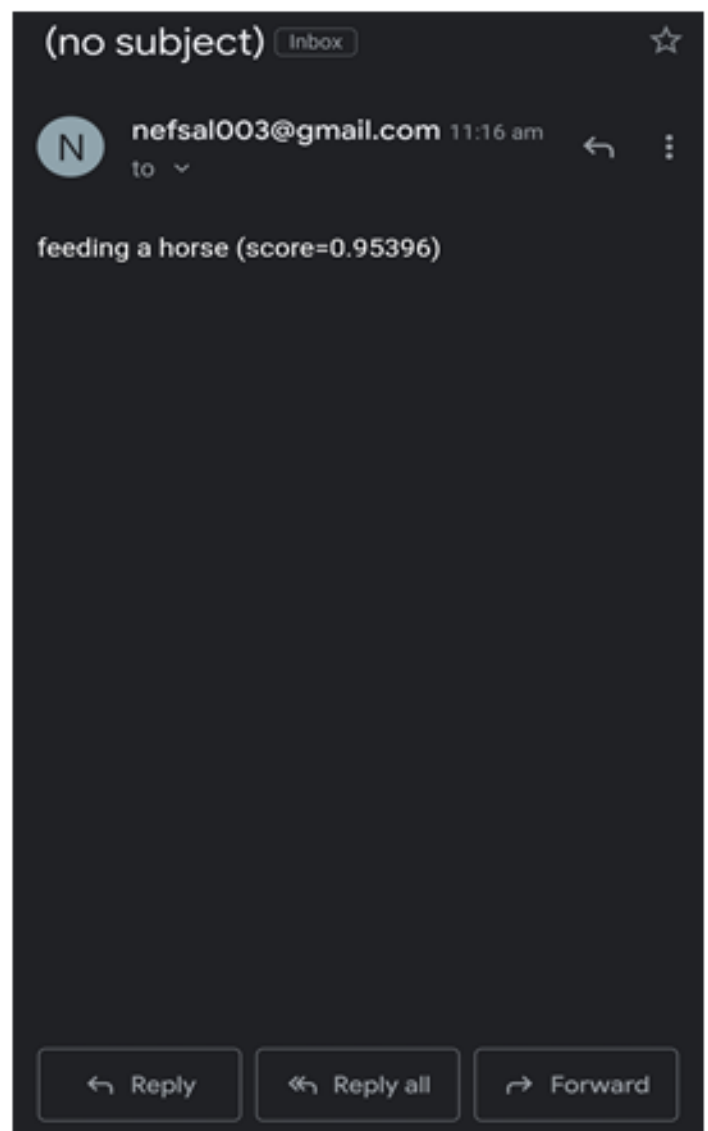

Fig 11:- Message2 sent

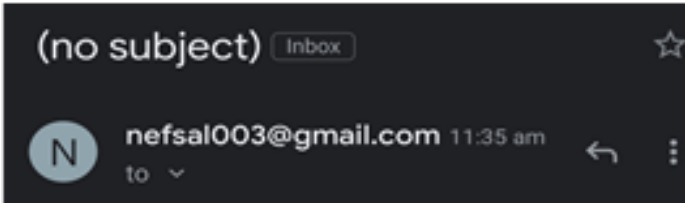

pushing a cart (score $=0.22414$ )

Fig 12:- Message3 sent 


\section{CONCLUSION}

Formal paid care services offered by caregivers or elderly care centers are expensive and thus are still out of reach for a large section of the elderly population living under constrained or fixed budget conditions. A robust fall detection system is essential to support the independent living of elderlies. Since we are using a camera to capture activities of aged person each and every movement of that person is captured. So, this will help to monitor individual apart from a hospital environment. The aged people may require frequent, immediate medical support, which may otherwise result into fatal consequences even death of the person. This system is essential to support the independent living of elderlies. Since we are using a camera to capture activities of aged person each and every movement of that person is captured. Also no need to wear any device in this case. So the disadvantages like fail to wear the device and charging failures can be avoided. Sometimes family members may send aged people to old age homes because they won't be able to take care of them because of their career challenges. So this system may help ages one's to remain themselves in a safe environment and if any care is needed then the corresponding person is getting a message.

\section{FUTURE ENHANCEMENTS}

Proposed project can be enhanced by expanding the dataset. Many improvements of this new approach are possible. Anomaly detection is an active area of current machine learning and data mining research. Anomaly detection methods can be applied to problems as diverse as monitoring of credit card transactions, detection of network intrusions, and detection of technical system failures. One can exploit the rich scope such insights has to offer in developing real time human asset monitoring in highly secured installations, tracking Elderly or population with movement disability or illness for any emergencies based on movement patterns, determining if a person id under fatigue or not and so on and so forth. The application domains are as broad as from healthcare to security services and fitness monitoring.

\section{REFERENCES}

[1]. P. Barralon, I. Dorronsoro, and E. Hernandez, "Automatic fall detection:Complementary devices for a better fall monitoring coverage," in Proc.IEEE 15th Int. Conf. e-Health Netw., Appl.

[2]. Zhen-Peng Bian, Junhui Hou, Lap-Pui Chau, and Nadia Magnenat-Thalmann, "Fall Detection Based on Body Part Tracking Using a Depth Camera" IEEE Journal of Health Informatics.

[3]. Li, X., Pang, T\& Wang, T. (2017). "Fall detection for elderly person care using convolutional neural networks." 2017 10th International Congress on Image and Signal Processing, BioMedical Engineering and Informatics.

[4]. Vallejo, M., \& Lopez, J. D. (2013). “Artificial Neural Networks as an alternative to traditional fall detection methods." 2013 35th Annual International Conference of the IEEE Engineering in Medicine and Biology Society.

[5]. Rougier, C., Meunier, J., St-Arnaud, A., \& Rousseau, J. (2011). "Robust Video Surveillance for Fall Detection Based on Human Shape Deformation." IEEE Transactions on Circuits and Systems for Video Technology, 21(5)

[6]. Anderson, D., Keller, J. M., Chen, X., \& He, Z. (2006). "Recognizing Falls from Silhouettes." 2006 International Conference of the IEEE Engineering in Medicine and Biology Society.

[7]. Karantonis, D. M., Narayanan, M. R., Mathie, M., Lovell, N. H., \& Celler, B. G. (2006). "Implementation of a Real-Time Human Movement Classifier Using a Triaxial Accelerometer". IEEE Transactions on Information Technology in Biomedicine, 10(1).

[8]. Naranjo-Hernandez, D., Roa, L. M., Reina-Tosina, J., \& Estudillo-Valderrama, M. A. (2012). "SoM: A Smart Sensor for Human Activity Monitoring and Assisted Healthy Ageing." IEEE Transactions on Biomedical Engineering, 59(11), 3177-3184.

[9]. Kostopoulos, P., Nunes, T., Salvi, K., Deriaz, M., \& Torrent, J. (2015). F2D: "A fall detection system tested with real data from daily life of elderly people." 2015 17th International Conference on E-Health Networking,

[10]. Hsu, S.-C., Chuang, C.-H., Huang, (2018). "A videobased abnormal human behavior detection for psychiatric patient monitoring." 2018 (IWAT). 